\title{
Alguns problemas em torno da categoria lexical verbo em línguas Tupí-Guaraní
}

\author{
Aryon Dall'Igna Rodrigues
}

Nesta comunicação são apontados alguns problemas que se apresentam no tratamento da categoria lexical verbo em línguas da família Tupí-Guaraní. Há um problema mais geral, que tem sido debatido ou tem estado implícito em muitos trabalhos nos últimos anos, que é o do reconhecimento de uma subcategoria lexical de verbos intransitivos estativos oposta a outra de verbos intransitivos ativos. Não vou retomar esse debate aqui, mas, atento-me a um critério mais morfológico que sintático, segundo o qual identifico predicados intransitivos possessivos (e atributivos), com núcleos nominais, distintos de predicados intransitivos estativos e ativos, com núcleos verbais (Rodrigues, 2000; cf. Dietrich, 2000), vou mencionar alguns problemas que, no seu conjunto, devem contribuir para a revisão da questão mais geral. O denominador comum desses problemas é a ocorrência dos sufixos -(r)amo e -(r)eme, considerados na identificação de formas gramaticais de uma mesma língua e também na comparação das diversas línguas. O primeiro problema levantado é posto pelas orações independentes iniciadas por complemento circunstancial, expressas, segundo a pessoa de seus respectivos sujeitos, de maneiras substancialmente diferentes das orações não iniciadas por tais complementos (1). O segundo problema diz respeito a essas mesmas orações, quando os núcleos de seus predicados são de natureza nominal, pois há línguas que marcam esses núcleos da mesma forma que os núcleos estritamente verbais, ao passo que a maioria delas os marca de maneira distinta, com o sufixo -(r)amo (2). O terceiro problema é o da forma que tomam os verbos nas orações subordinadas (no chamado modo subjuntivo) com sujeito diferente do da oração principal, em algumas línguas com o sufixo -(r)eme, em outras com -(r)amo (3). Um quarto fato a ser observado na morfologia do verbo é o gerúndio, que marca a identidade de sujeitos no sistema de referência alternada ou switch reference: nos verbos intransitivos e transitivos é marcado por reflexos do su- 
fixo proto-Tupí * -áßo $\sim^{*}-a$, mas nos núcleos nominais de predicados ocorre, em lugar desses, o sufixo -(r)amo (4). Por fim, nessa constelação de formas que têm como denominador morfológico comum o(s) sufixo(s) de forma -(r)amo, deve ser considerado ainda o caso nominal translativo, cujo sufixo tem essa mesma forma (5).

\section{Orações independentes com circunstância topicalizada}

Quando uma oração independente contém um complemento circunstancial e este é topicalizado e colocado no início da oração, precedendo todos os seus outros constituintes, o núcleo do predicado pode tomar uma forma diversa da que tem nas orações independentes em que não se dá essa topicalização. Essa forma é marcada por um sufixo modal específico, o qual tem sido tratado como marca de um modo indicativo II (Rodrigues 1953, p. 126, Almeida et al. 1983, p. 34, Vieira \& Leite 1998, p. 29) ou modo circunstancial (Rodrigues 1981, Jensen 1990, p. 105, Praça 2000, p. 560, Seki 2000, p. 26). À diferença do modo indicativo I das orações independentes sem topicalização de complemento circunstancial, o indicativo II não recebe flexão pessoal, mas recebe, em seu lugar, flexão relacional. Esta última consiste basicamente em marcar por prefixos a dependência de um elemento determinado, neste caso o núcleo do predicado, em relação ao seu determinante imediato, que é o objeto dos predicados transitivos e o sujeito dos predicados intransitivos (Rodrigues, 1996, p. 58).

O contraste entre o indicativo II e o indicativo I pode ser ilustrado pelos seguintes exemplos do Tupinambá, com predicados transitivos (1a-b e 3a-c) e intransitivos $(2 a-d$ e $4 a-c):{ }^{1}$

(1a) apiáß-a oje?i tapi?ír-a o-s-epják

(homem-ARg hoje.passado anta-ARg 3sUJ-REL.N-ver)

"um homem viu hoje uma anta"

1. Abreviaturas usadas nas glosas: ARG: sufixo do caso argumen(ta)tivo, AUM: sufixo aumentativo, cAUS: causativo, CAUS.CoM: causativo-comitativo, FUT: futuro, GER: gerúndio, HIP: hipotético, IND.I: modo indicativo I, IND.II: sufixo do modo indicativo II, IND: intencional, Loc.PONT: sufixo do caso locativo pontual, Loc.sit: sufixo do caso locativo situacional, REFL: reflexivo, REL.C: prefixo relacional de contiguidade do determinante, REL.H: prefixo relacional de determinante humano indefinido, REL.N: prefixo relacional de não contiguidade do determinante, SUBJ: subjuntivo, sUJ: sujeito, TRANSL: caso translativo, 3c: 3a. pessoa co-referente, 3s: 3a. pessoa sujeito. 
(1b) ojeßí apíáß-a tapi?ír-a r-epják-i

(hoje homem-ARG anta-ARG REL.C-ver-IND.II)

"hoje um homem viu uma anta"

(2a) $\quad a$-júr ók-a suí < Aiûr òca çuî>

(1suJ-vir IND.I casa-ARG de)

"venho de casa" (Anchieta, 1595, p. 40)

(2b) mosapír aßá-Ø 0 -úr < moçapîr abâ oûr >

(três pessoa-ARg 3s-vir IND.I)

"três pessoas vieram" (Anchieta, 1595, p. 9v)

(2c) kó sjé Ø-anám-a r-úr-i pá

(aqui eu REL.C-parente-ARG REL.C-vir-IND.II)

"aqui vieram todos os meus parentes" (Anchieta, 1954, p. 84)

(2d) sjé Ø-pitá-j $t$-úr-i<xepitâi túri>

(eu REL.C-calcanhar-LOC.SIT REL.C-vir-IND.II)

"no meu calcanhar veio (i. é, detrás de mim)"

(Anchieta, 1595, p. 41v)

(3a) sjé oro-juká

(eu 2OBJ-matar IND.I)

"eu te mato" (Anchieta, 1595, p. 37)

(3b) koromõ sjé né Ø-juká-w < coromóxendejucáu>

(logo eu tu REL.C-matar-IND.II)

"logo eu te mato" (Anchieta, 1595, p. 39v)

(3c) korí Pedro oré Ø-juká-w< corí pedro orejucáo>

(hoje FUturo Pedro nós EXcLusivo REL.C-matar-IND.II)

"hoje Pedro nos (excl.) mata" (Anchieta, 1595, p.39v)

(4a) $\quad a$-só <açô>

(1sUJ-ir IND.I)

"eu vou" (Anchieta, 1595, p. 39v)

(4b) koromõ sjé $\varnothing$-só-w < coromôxeçou>

(logo eu REL.C-ir-IND.II)

"logo vou" (Anchieta, 1595, p. 39v) 
(4c) kwesé Pedro Ø-só-w < Quecé Pedro çóu> (ontem Pedro REL.C-ir-IND.II)

"Ontem Pedro foi" (Figueira, 1687, p. 95)

Em grande parte das línguas TG o modo indicativo II se restringe a predicados cujo sujeito é de terceira pessoa (Asuriní do Tocantins, Asuriní do Xingu, Suruí, Tapirapé, Guajajára, Parintintín, Guaráyo), mas em algumas ele compreende também predicados com sujeitos de primeira pessoa (Tupinambá, Guaraní antigo, Kayabí) e em Araweté ocorre também com sujeitos de segunda pessoa (Vieira \& Leite 1998, p. 29).

Os seguintes são exemplos do Asuriní do Xingu (5) e do Guaraní antigo (6):

(5a) a-kupit aite

(1suJ-roçar IND.I sempre)

"roço sempre" (Monserrat et al., 1988, p. 21)

(5b) aite ae Ø-kupir-i

(sempre a gente REL.C-roçar-IND.II)

"sempre a gente roça" (Monserrat et al., 1988, p. 21)

(6a) kóramonatú t fe $r$-úr-i<corãmõngatú cherúri>

(agora eu REL.C-vir-IND.II)

"agora venho" (Ruiz de Montoya, Tesoro 96)

(6b) kó-ßét tee r-ú-j<cobé cheruî>

(aqui-ainda eu REL.C-estar.deitado-IND.II)

"aqui estou ainda" (Ruiz de Montoya, Tesoro 7v)

\section{O Indicativo II com predicados de núcleo nominal}

Nos exemplos 1 a 4 acima o sufixo do modo indicativo II tem dois alomorfes, $-i$ após temas terminados em consoante e $-w$ após temas terminados em vogal. Num dialeto do Tupinambá, aquele que é representado pela gramática de Figueira (1621) e que é do norte do Brasil, esse sufixo ocorre tanto com os núcleos verbais transitivos (1b, 3b, 3c) e intransitivos (2c, 2d, 4b, 4c) como com os núcleos nominais dos predicados ( $7 \mathrm{~b}, 8 \mathrm{~b}$, abaixo):

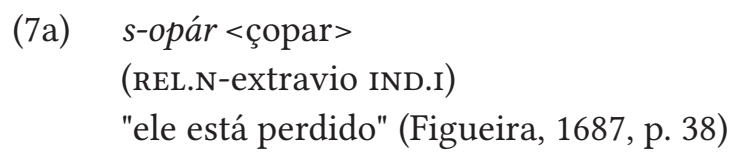


(7b) kwesé ka?á-Ø r-upí Pedro o-watá- $\beta$ o s-opár-i

(ontem mato-ARG REL.C-por Pedro 3C-andar-MS REL.N-extravio-IND.II) "ontem Pedro se perdeu andando pelo mato" (Figueira, 1687, p. 95)

(8a) i-ma?ẽnwár <imäenduár>

(REL.N-lembrança IND.I)

"ele se lembra" (Figueira, 1687, p. 36)

(8b) kwesé Pedro né r-esé i-ma?ẽn wár-i <quecé Pedro nderecé imaenduári>

(ontem Pedro você REL.C-acerca.de REL.N-lembrança-IND.II)

"ontem Pedro de ti se lembrou" (Figueira, 1687, p. 95)

Entretanto, em outro dialeto da mesma língua, o da gramática de Anchieta (1595), que é da costa leste do Brasil, os núcleos nominais no indicativo II são marcados pelo sufixo -amo -ramo:

(9a) $s$-orí $\beta<$ çorîb $>$

(REL.N-alegria IND.I)

"ele se alegrou" (Anchieta, 1595, p. 38)

(9b) koromõ sjé $r$-orí $\beta$-amo <coromoxeroríbamo>

(logo eu REL.C-alegria-IND.II)

"logo eu me alegro" (Anchieta, 1595, p. 40)

Como a gramática de Anchieta terá sido organizada inicialmente com base na língua Tupí de São Vicente e Piratininga, tendo sido depois adaptada para o Tupinambá da costa leste, o que é denunciado por diversas inconsistências na sua exemplificação, pode-se pensar que a diferença no indicativo II dos núcleos nominais em Anchieta seja mais uma característica do Tupí que teria escapado à adaptação do livro para o Tupinambá. ${ }^{2}$

As demais línguas da família para as quais há documentação disponível comportam-se neste particular como o que foi descrito e exemplificado por

2. Outra característica do Tupí que transparece nos exemplos da Arte de Anchieta é o alomorfe $\varnothing$ - do prefixo relacional de não contiguidade fazendo referência ao objeto no indicativo I dos verbos causativos em vez do alomorfe $j$ do Tupinambá: aimondô (a-j-mosó) $(1595$, p. 1) e amondô (a-Ø-mo-só) (1595, p. 3v) "eu o faço ir", aimonguér (a-j-mo-kér) (1595, p. 5) "eu o faço dormir" e amongúb (a-Ø-mo-kúb) (1595, p. 5) "eu os faço estar", aimogebîr (a-j-mo-jebttr) (1595, p. 48) "eu o faço voltar" e amogebîr (a-Ø-mo-jebtir) (1595, p. 48v) (cf. Rodrigues 1997, p. 375, n. 4). 
Anchieta, de modo que o Tupinambá descrito por Figueira é único em usar o mesmo sufixo de modo para os núcleos verbais e nominais no indicativo II. Uma hipótese para essa discrepância entre o dialeto setentrional do Tupinambá e o dialeto oriental e as demais línguas da família, é a de que estes últimos representem mais diretamente a situação original na proto-língua da família e que o dialeto setentrional tenha estendido por analogia o sufixo modal dos núcleos verbais aos núcleos nominais. Para essa extensão analógica pode ter contribuído a homonímia do sufixo dos núcleos nominais no modo indicativo II com as demais ocorrências do(s) sufixo(s) -(r)amo, a qual assim teria sido reduzida.

Exemplos do indicativo II dos núcleos nominais em algumas outras línguas da família:

\section{Kayabí}

ka1á-Ø r-upí je Ø-ó-j

(mata-ARG REL.C-por eu REL.C-ir-IND.II)

"fui pela mata" (Dobson 1988, p. 69)

(11) pẽ Ø-pir-i je r-úr-i

(vocês proximidade-LOC.SIT eu REL.C-vir-IND.II)

"vim visitar vocês" (Dobson, 1988, p. 76)

Tapirapé

(12) kwãxí-Ø r-ewír-i i-ij-Ø xe r-eimãw-ã

(quati-ARG REL.C-traseiro-LOC.SIT REL.N-correr-IND.II eu REL.C-xerimbabo-ARG)

"meu xerimbabo (o cachorro) correu atrás do quati"

(arquivo de campo de W. N. Praça)

(13) $\quad$ 2ý-Ø r-opí i-xãók-i t-amõj-ã

(rio-ARG REL.C-por REL.N-banhar.se-IND.II REL.H-avô-ARG)

"pelo rio está banhando-se um avô"

(arquivo de campo de W. N. Praça)

A existência do indicativo II e as restrições a que está sujeito segundo as pessoas do discurso, constituem problemas a ser pensados, tanto de um ponto de vista funcional, quanto comparativa e diacronicamente dentro do tronco linguístico Tupí e, mais especificamente, dentro da família Tupí-Guaraní. O fato de que o indicativo II não seja construído com prefixos flexionais de 
pessoa, mas só com prefixos relacionais, parece apontar para um desenvolvimento histórico a partir de uma construção nominal. Essa possibilidade é reforçada pela ocorrência do sufixo -(r)amo, como se verá adiante.

\section{Modo subjuntivo}

As orações subordinadas condicionais e temporais (orações se/quando) são expressas pela forma verbal que se convencionou chamar de modo subjuntivo. Em Tupinambá esse modo é marcado pelo sufixo -reme -eme -me, comum às duas variedades, oriental e setentrional. O sufixo é essencialmente o mesmo em Wayampí e é semelhante em Asuriní do Xingu e em Parintintín (-rame -ame) e Tembé (-mehe), mas é homônimo do caso argumen(ta)tivo em Guaraní, Tapirapé, Asuriní do Tocantins, Kayabí. Exemplos:

\section{Tupinambá}

(14) sjé Ø-mo-nó-reme = mo $a$-só=mo < xémondórememô açômo > (eu REL.C-CAUS-ir-SUBJ=HIP $1 \mathrm{~s}$-ir=HIP) "se me mandaram, iria eu" (Anchieta, 1595, p. 25)

(15) sjé ma?é-?ú sjé r-én-eme <xembaeú xeréneme> (eu coisa-ingerir IND.I eu REL.C-estar.sentado-SUBJ) "quando eu estava comendo" (Anchieta, 1595, p. 26v)

(16) Pedro já?wár-a Ø-juká-reme < Pedro iaguára iucáreme> (Pedro onça-ARG REL.C-matar-SUBJ) "se Pedro matar a onça" (Figueira, 1687, p. 155)

(17) sjé Ø-ma7ẽnwár-eme <xemäenduáreme> (eu REL.C-lembrado-sUBJ) "quando me lembro, se me lembro" (Figueira, 1687, p. 47)

\section{Asuriní do Xingu}

(18) pajé-Ø te-kutúk-ame n a-ja?á-j<paje te kutukame naja'ai> (pajé-ARG 1C-furar-sUBJ não 1s-chorar IND.I -NEG) "quando o pajé me furou, não chorei" (Monserrat et al., 1988, p. 30).

(19) je r-̃̃j-a Ø-at́t-rame $a$-ja?á <je rỹjá ayrame aja'a> (eu REL.C-dor-SUBJ 1s-chorar IND.I) "quando meu dente doeu eu chorei" (Monserrat et al., 1988, p. 30) 


\section{Guaraní antigo}

(20) t té o-mo-?é-ramo o-j-kwaá <Che omboérãmõ oiquaà> (eu 3C-CAUS-dizer-SUBJ 3C-REL N-saber IND.I) "se eu lhe ensinasse, ele saberia" (Ruiz de Montoya, Arte 22)

(21) $a$-h-aihú t é $r$-aithú-ramo=ne < Ahaŭhú cheraŭhúrãmõne> (1s-REL N-amar IND.I eu REL.C-amar- SUBJ=IND) "eu o amarei se ele me amar" (Ruiz de Montoya, Arte 20)

\section{4. $\mathrm{O}$ gerúndio}

A forma que toma o verbo numa oração cujo sujeito é co-referente do sujeito ou do objeto de outra oração da qual ela depende ou com a qual ela se coordena, tem sido chamada de gerúndio na longa tradição - mais de quatrocentos anos - de descrição das línguas da família Tupí-Guaraní. O gerúndio dos núcleos verbais de predicados intransitivos e transitivos é formado por reflexos do sufixo *-áßo * - $a$ do Proto-Tupí-Guaraní, ao passo que o dos núcleos nominais se faz com o sufixo -(r)amo, ambos indicando mesmo sujeito. Exemplos:

\section{Tupinambá}

(22) o-só Pedro ja?wár-a Ø-juká- $\beta$ o <oçó Pedro iaguára iucâbo> (3s-ir IND.I Pedro onça-ARg REL.C-matar-GER)

"foi Pedro a matar a onça" (Figueira, 1687, p. 155)

(23) kwesé ka?á-Ø r-upí o-watá-ßo Pedro r-opár-i

<quecé cäá rupi oguatábo Pedro ropári>

(ontem mato-ARG REL.C-por 3s-andar-GER Pedro REL.C-extravio-IND.II)

"ontem, andando pelo mato, Pedro se perdeu" (Figueira, 1687, p. 95)

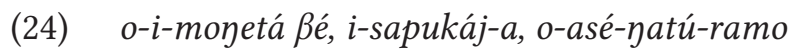

< oimong-etábê, oçapucaya oçacêgaturamo>

(3S-REL.N-falar com IND.I também, REL.N-clamar-GER, 3C-gritar-bem-GER)

"também falou com ele, clamou e gritou muito" (Araújo, 1618, p. 63v; trad. de ADR)

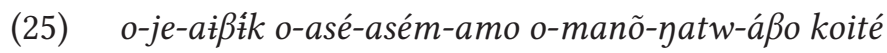
<oyeaîbic ogoacè acemamo omanôgatuâbo coite> 
(3C-REFL-abaixar a cabeça IND.I 3C-gritar-gritar-GER 3s-morrer-bem-GER enfim)

"abaixou a cabeça, gritou repetidamente e enfim morreu mesmo" (Araújo, 1618, p.63v; trad. de ADR)

\section{Asuriní do Xingu}

(26) itá-Ø u-pițk muité i-ma-má-ta <ita upyyk, muite imamata> (pedra-ARg 3s-pegar IND.I longe REL.N-CAUS-pular-GER) "pegou a pedra e jogou-a longe" (Monserrat et al., 1988, p. 17)

(27) $u$-úß-a r-upí i-á-Ø u-atá-w <uuva rupi ia uatau> (3C-pai-ARG REL.N-ir-IND.II 3C-caçar-GER) "foi caçar com o pai" (Monserrat et al., 1988, p. 17)

(28) ipirá- $\varnothing$ u-enu-ém $\varnothing$-eý-ta i-?ú- $\varnothing<$ ipira uenuem, eyta, i'u> (peixe-ARG 3S-CAUS.COM-sair IND.I REL.N-assar-GER REL.N-comer-GER) "pegou peixe, assou-o, comeu-o" (Monserrat et al., 1988, p. 16)

\section{5. $O$ caso translativo}

Há na família linguística Tupí-Guaraní um sistema de casos morfológicos, mais desenvolvido em algumas línguas, mais limitado em outras. Em Tupinambá, que tem um sistema mais rico, há para os nomes um caso reto e quatro casos adverbiais (os pronomes pessoais não recebem as marcas desses casos, mas, sim, a do caso dativo, que nos nomes só é expresso por meio de posposição (Rodrigues, 2000)). O caso reto é o argumen(ta)tivo, próprio dos argumentos principais, isto é, tanto dos sujeitos e objetos diretos, quanto dos objetos de posposições (Rodrigues, 1996). Os casos adverbiais são o translativo, o locativo pontual, o locativo difuso e o situacional (Rodrigues, 2000). O caso translativo, que indica a mudança de um ator ou de um objeto de um estado para outro ou de uma situação para outra, é expresso por um sufixo homônimo daqueles que exprimem o modo indicativo II e o gerúndio dos predicados com núcleo nominal: -amo -ramo. Exs.:

\section{Tupinambá}

(29) isé $t$-ú $\beta$-amo $a$-ikó=ne <ixe túbamo aicóne> (eu REL.N-pai-TRANSL 1sUJ-estar IND.I=INT) "serei como um pai para ele" (Figueira, 1687, p. 121) 
(30) sjé Ø-irũ-namo s-ekó-w <xeyrúnamo cecóu>

(eu REL.C-companheiro-TRANSL REL.N-estar-IND.II)

"está comigo" (Figueira, 1687, p. 123)

\section{Asuriní do Xingu}

je tipé inimá-Ø Ø-jár-amu

(eu somente linha-ARG REL.C-dono-TRANSL)

"só eu sou (como) dono da linha" (Monserrat et al., 1988, p. 33)

(32) yaký-Ø u-mu-'ít-atá-rúm-amu

(lenha-ARG 3SUJ-CAUS-pequeno IND.I REL.H-fogo-FUT-TRANSL)

"cortou lenha para (servir como [futuro]) fogo" (Monserrat et al., 1988, p. 34)

\section{Breve discussão}

Os fatos expostos acima põem vários problemas tanto para a análise das línguas da família Tupí-Guaraní, como para a reconstrução do desenvolvimento histórico das mesmas. Além da questão mais geral da caracterização tipológica destas línguas, já referido no início deste trabalho, há diversos problemas com diferentes graus de especificidade, isto é, alguns encontrados numa só língua, como o do sufixo do indicativo II em Tupinambá (1 e 2), outros distinguindo grupos de línguas, como as formas do sufixo do subjuntivo divididas entre -(r)eme e -(r)amo (3), outros ainda comuns a toda a família, como o da natureza e origem do indicativo II e o da natureza do gerúndio (4). Fenômenos ocorridos diacronicamente como analogia, nivelamento paradigmático, anti-homonímia e outros devem ter concorrido para as atuais semelhanças e diferenças e devem ser detectados para a reconstrução do estado linguístico de que se derivaram as línguas da família, isto é, do Proto-Tupí-Guaraní. Alguns desses fenômenos não são exclusivos desta família, mas ocorrem ou têm correspondências em línguas de outras famílias do tronco linguístico Tupí, de modo que sua melhor compreensão dependerá também do conhecimento das situações dessas. Além da homonímia do sufixo nominal do caso translativo -(r)amo (5) com os sufixos que marcam o indicativo II e o gerúndio dos núcleos nominais de predicados e, em parte das línguas da família, com aquele que marca o subjuntivo, há uma outra forte aproximação dessas formas modais com os nomes, já que nenhuma delas tem flexão pessoal, mas só têm flexão relacional, tal como os nomes, quando o seu determinante é diferente do sujeito da respectiva oração. 


\section{Referências}

Almeida, Antônio, et al, 1983, A língua Tapirapé. Rio de Janeiro: Xerox.

Anchieta, José de, 1595, Arte de grammatica da lingua mais usada na costa do Brasil. Lisboa: Antonio de Mariz, (Diversas reedições facsimilares: Leipzig: Teubner, 1876; Rio de Janeiro: Bibliotheca Nacional, 1933; São Paulo: Anchietana, 1946; Salvador: Universidade Federal da Bahia, 1980 e 1981; São Paulo: Loyola, 1990.) , 1954, Poesias. Manuscrito do séc. XVI, em português, castelhano, latim, e tupi. Transcrições, traduções e notas de M. de L. de Paula Martins. Boletim IV, Documentação Lingüística 4, do Museu Paulista. São Paulo: Comissão do IV Centenário da Cidade de São Paulo (Reprodução fac-similar: Belo Horizonte/São Paulo, 1989.)

Araujo, Antonio de, 1618, Catecismo na língoa brasilica. Lisboa: P. Cras-beeck (Reprodução facsimilar: Rio de Janeiro: Pontifícia Universidade Católica do Rio de Janeiro, 1952).

Dietrich, Wolf, 2000, Categorias lexicais nas línguas tupi-guaranis (visão comparativa). In: QUEIXALÒS, F.(org.). Des noms et des verbes en Tupi-Guarani, état de la question. Caiena: IRD e CNRS, p. 1-13.

Dobson, Rose M, 1988, Aspectos da língua Kayabí. Brasília: Summer Institute of Linguistics (Série Lingüística, 12).

Figueira, Luis, 1687, Arte de grammatica da lingua brasilica. Lisboa: M. Deslandes (Reedição facsimilar: Leipzig: Teubner, 1878.).

Jensen, Cheryl Joyce S., 1990, O desenvolvimento histórico da língua Wayampi. Campinas: Editora da UNICAMP.

Monserrat, Ruth Maria Fonini, e Irmãzinhas de Jesus. Língua Asurini do Xingu: observações gramaticais. Altamira (Pará): Conselho Indigenista Missionário, (1988).

Praça, Walkíria Neiva, 1999, Nomes como predicados em Tapirapé. Dissertação de mestrado, Universidade de Brasília.

Rodrigues, Aryon Dall'Igna, 1953, Morfologia do verbo em Tupi, em Letras. Curitiba, n. 1, p. 121-152.

1981, "Estrutura do Tupinambá", Trabalho não publicado.

, 1996, Argumento e predicado em Tupinambá. ABRALIN, Boletim da Associação Brasileira de Linguística. Maceió, n. 19, p. 57-66.

2000, Sobre a natureza do caso argumentativo. In: QUEIXALÒS, F.(org.). Des noms et des verbes en Tupi-Guarani, état de la question. Caiena: IRD e CNRS, p.63-74.

Ruiz de Montoya, Antonio, 1639. Tesoro de la lengua guarani. Madrid. (Reedição facsimilar: Leipzig: Teubner, 1876; reedição não facsimilar: Viena: Faesy \& Frick, Paris: Maisonneuve 1876).

1640, Arte, y Bocabulario de la lengua guarani. Madrid: Juan Sanchez (Reedições facsimilares: Leipzig: Teubner, 1876, e Madrid: Cultura Hispánica, 1994; reedição não facsimilar: Viena: Faesy \& Frick, Paris: Maisonneuve 1876). 
Seki, Lucy, 2000, Classes de palavras e categorias sintático-funcionais em Kamaiurá. In: QUEIXALÒS, F. (org.). Des noms et des verbes en Tupi-Guarani, état de la question. Caiena: IRD e CNRS, p. 14-40.

Vieira, Márcia Damaso; Leite, Yonne de Freitas, 1998, Observações preliminares sobre a língua Araweté. Moara, Revista dos Cursos de Pós-Graduação em Letras. Belém, n. 9, p. 7-31, jan./jun. 